\title{
SENSIBILIDAD Y ESPECIFICIDAD DEL CUESTIONARIO DE PREOCUPACIÓN Y ANSIEDAD PARA LA DETECCIÓN DEL TRASTORNO DE ANSIEDAD GENERALIZADA EN LA EDAD AVANZADA
}

\author{
ROBERTO NUEVO \\ Universidad Autónoma de Madrid \\ (Aceptado en marzo de 2005)
}

\begin{abstract}
Este trabajo se dirige a comprobar, mediante el análisis de las curvas COR (características operativas del receptor), la capacidad para identificar la presencia de trastorno de ansiedad generalizada (TAG) en personas mayores del Cuestionario de Preocupación y Ansiedad (WAQ), una medida dimensional de los criterios diagnósticos del TAG según el DSM-IV. La muestra estuvo compuesta por 97 personas entre 65 y 90 años (media $=73,4 ; \mathrm{DT}=5,6)$. La presencia de TAG $(\mathrm{n}=7)$ se estableció mediante una entrevista diagnóstica estructurada. Una puntuación igual o superior a 38 maximizó simultáneamente la sensibilidad (100\%) y la especificidad $(91,1 \%$ ) de la escala para la identificación del TAG. Los resultados de este trabajo ofrecen apoyo adicional a la fiabilidad y validez del WAQ en su uso con personas mayores, dado que presenta sensibilidad y especificidad elevadas en la identificación del TAG. Esto permite sugerir la utilidad del empleo de esta escala y de los puntos de corte ofrecidos por las curva COR, como un medio de identificación y cribado del TAG en esta edad.
\end{abstract}

Palabras clave: Ansiedad, cribado, análisis COR, personas mayores, diagnóstico

Sensitivity and specificity of the Worry and Anxiety Questionnaire for detecting generalized anxiety disorder in the old age

This work aimed to test, through the analysis of the ROC (receiver operating characteristics) curves, the ability of the Worry and Anxiety Questionnaire (WAQ) to identify the presence of generalized anxiety disorder (GAD) in older adults. Sample was composed by 239 subjects between 65 and 90 years old (Mean $=73,4 ; \mathrm{SD}=5,6$ ). The presence de GAD $(n=7)$ was established with a structured interview. A cut score of 38 (equal or higher) maximized both sensitivity (100\%) and specificity $(91,1 \%)$ for identifying GAD. Results of this work provide additional support to the validity and reliability of WAQ with older adults, given that it presents high sensitivity and specificity to identify the presence of GAD. This allows suggest the usefulness of this scale and its cut-score according to the ROC curve like instruments for the identification and screening of GAD in the late life.

Key words: Anxiety, screening, ROC analysis, older adults, diagnosis

\section{INTRODUCCIÓN}

El trastorno de ansiedad generalizada (TAG) tiene una elevada prevalencia en la edad avanzada. Según la revisión de

Correspondencia: Roberto Nuevo, Departamento de Psicología Biológica y de la Salud, Facultad de Psicología, Universidad Autónoma de Madrid, Ciudad Universitaria de Cantoblanco, 28049 Madrid. Correo-e: roberto.nuevo@uam.es
Flint (1994), entre un $0,71 \%$ y un $7,1 \%$ de personas mayores cumple los criterios diagnósticos del DSM-IV (APA, 1994) para el TAG. Resultados similares, $7,2 \%$, han sido encontrados en población mayor española (Montorio, Nuevo, Losada y Márquez, 2001). También, diferente información indirecta sugiere que la relevancia de este trastorno en esta edad es incluso superior a la indicada 
por los datos epidemiológicos. Así, por ejemplo, Himmelfarb y Murrell (1984), encontraron que casi un $20 \%$ de personas mayores presentaba niveles de ansiedad-rasgo en un nivel clínico o subclínico de acuerdo con sus puntuaciones en la versión rasgo del STAI. Por otra parte, el TAG está asociado a elevados costes en los servicios de salud (Greenberg et al., 1999), así como a un importante deterioro de la calidad de vida de las personas mayores (Stanley y Beck, 2000; Wetherell et al., 2004) y parece ser el síntoma más frecuentemente informado en las consultas de atención primaria (Goldberg, Bridges, Duncan-Jones y Grayson, 1987). Estos datos recalcan la importancia de desarrollar medidas para la detección rápida y eficaz de posibles casos de TAG en la edad avanzada. Por otra parte, aunque se han señalado algunas diferencias en las características de la preocupación excesiva atribuibles al envejecimiento, por ejemplo en los contenidos en los que se centra la preocupación (Nuevo, Izal, Montorio, Losada y Márquez, 2003), alguna evidencia empírica preliminar mediante análisis factorial confirmatorio permite sostener la idea de que la estructura factorial de la sintomatología del TAG es invariante entre muestras de personas mayores y estudiantes universitarios (Nuevo et al., manuscrito enviado).

El objetivo de este trabajo es analizar la capacidad para identificar la presencia de TAG en población de edad avanzada de un instrumento que evalúa de manera dimensional los criterios diagnósticos del DSM-IV para el TAG. A diferencia de otros instrumentos similares que preguntan por cada uno de los síntomas o criterios de manera dicotómica para entonces aplicar los algoritmos del DSM-IV para determinar la presencia de cada trastorno, el Cuestionario de Preocupación y Ansiedad (Worry and Anxiety Questionnaire, WAQ; Dugas et al., 2001) asume una concepción dimensional del trastorno y pregunta por el grado de intensidad de cada síntoma en una escala 0-8. Aunque los autores del WAQ proponen un algoritmo para la identificación del TAG estableciendo un punto de corte de 5 o más para cada síntoma y aplicando entonces el algoritmo del DSM-IV igual que si las puntuaciones fueran dicotómicas, este punto de corte es necesariamente arbitrario $y$, sobre todo, suprime la ventaja potencial de una medida dimensional de los criterios: para tomar una decisión dicotómica sería más correcto preguntar directamente de manera dicotómica. Existe evidencia empírica desde aproximaciones taxométricas, sin embargo, de que el TAG podría funcionar de una manera dimensional (Ruscio, Borkovec y Ruscio, 2001) y de que cada uno de los síntomas está presente en algún grado en todas las personas, con o sin trastorno, por lo que una evaluación del grado de severidad de los síntomas del TAG sería más adecuada que la mera constatación de la presencia o no del síntoma. En este sentido, una alternativa al uso de pruebas dicotómicas como medio de cribado (screening) para la presencia de TAG es el uso de una medida dimensional como el WAQ y el establecimiento de puntos de corte para identificar la presencia del trastorno, lo que constituye el objetivo del presente trabajo.

Para ello se utilizó el análisis de la curva característica operativa del receptor (COR, traducción de las siglas inglesas ROC; receiver operating characteristics). $\mathrm{El}$ análisis COR se desarrolló en el contexto de la teoría de detección de señales como un medio de optimizar la decisión sobre si una determinada señal está o no presente. Aunque sus aplicaciones iniciales se centraron en el campo de la ingeniería y la psicofísica, eEn los últimos años se ha generalizado su uso en el ámbito clínico como medio de analizar la 
capacidad de una prueba para identificar la presencia o ausencia de un determinado trastorno (p. ej., Shapiro, 1999). El análisis COR permite conocer la capacidad de un cuestionario para identificar casos positivos y negativos de un grupo determinado, es decir, en el ámbito aplicado aporta información objetiva sobre la capacidad de una prueba como instrumento de cribado para la presencia de trastornos. En concreto, el análisis COR ofrece una curva que presenta gráficamente un eje con la sensibilidad (la probabilidad de identificar correctamente la presencia del trastorno) y otro eje con 1 menos la especificidad (la probabilidad de identificar correctamente la ausencia del trastorno).

Así, el análisis ofrece datos sobre la sensibilidad y especificidad asociada a cada puntuación en el instrumento de evaluación. Esto permite establecer cuantitativamente un punto de corte para la identificación de casos clínicos. Aunque varios criterios se han empleado para tomar la decisión de cuál es el punto de corte más adecuado, el Índice de Youden (Youden, 1950) es el más usualmente informado en la literatura. Este índice viene dado por el valor obtenido por la fórmula [(sensibilidad + especificidad) 1]. Los valores cercanos a 1 indican que tanto la sensibilidad como la especificidad asociadas a esa puntuación son elevadas. Otro índice de la bondad de una prueba como instrumento de cribado es el área bajo la curva, cuyos valores oscilan entre 0 y 1 . Puntuaciones superiores a 0,50 indican que la prueba clasifica correctamente más casos de los esperables por azar y valores cercanos a 1 indican un buen funcionamiento del instrumento. Una ventaja adicional del análisis COR es que la prueba es bastante robusta incluso cuando no puede asumirse la normalidad de las puntuaciones o las muestras son de tamaños diferentes (Rice y Harris, 1995).

\section{MÉTODO}

\section{Participantes}

La muestra inicial estuvo compuesta por 109 personas entre 65 y 90 años, de las cuales el 56,7 \% eran mujeres (media de edad $=74,0 ; \mathrm{DT}=5,9$ ) y el $43,3 \%$ eran hombres (media de edad $=72,6$; DT $=5,2$ ). El 26,8 \% había realizado estudios superiores, el $16,5 \%$ estudios secundarios, el 49,5\% estudios primarios y el 7,2 $\%$ no tenía ninguna formación académica. El 61,9\% estaban casados, el $34 \%$ viudos, el 3,1\% solteros y el $1 \%$ divorciados o separados. El Mini-Examen Cognoscitivo (versión de Lobo, Ezquerra, Gómez, Sala y Seva, 1979) se empleó como medio de detectar un posible deterioro cognitivo. Empleando un punto de corte de 25 (Del Ser y Peña-Casanova, 1994), 12 personas fueron excluidas de la muestra, que finalmente estuvo compuesta por 97 personas.

\section{Instrumentos}

El Cuestionario de Preocupación y Ansiedad (WAQ, Worry and Anxiety Questionnaire; Dugas et al., 2001), evalúa dimensionalmente (0-8) el grado en el que se presentan los diferentes criterios diagnósticos del DSM-IV para el TAG. Específicamente, cuatro ítems evalúan el grado en el que se presentan los cuatro criterios cognitivos del DSM-IV para el TAG (falta de control percibido sobre las preocupaciones, grado en el que son percibidas como excesivas, interferencia producida por la preocupación en la vida cotidiana y frecuencia de preocupación excesiva) y seis ítems evalúan los criterios de tipo somático o síntomas asociados al trastorno: inquietud o impaciencia, fatigabilidad fácil, dificultades para concentrarse o poner la mente en blanco, irritabilidad, tensión muscular y problemas para dor- 
mir (dificultad para quedarse dormido o sensación de sueño no reparador). La validación española del WAQ (Ibáñez et al., 2000), con una muestra de personas entre 18 y 88 años (Media = 29,10; DT $=10,84$ ), demostró que el cuestionario presentaba buenas propiedades psicométricas, con una elevada consistencia interna $(0,90)$ y fiabilidad test-restest $(0,80)$, validez convergente y discriminante y una estructura bidimensional, con los síntomas cognitivos (dimensión etiquetada por los autores como "preocupación») por un lado y los somáticos (dimensión etiquetada como emocionalidad) por el otro. En todo caso, la elevada correlación encontrada en ese trabajo entre los dos factores $(0,72)$ permite pensar en una posibilidad no analizada por los autores en su análisis factorial confirmatorio: la presencia de un factor de segundo orden que explique simultáneamente ambas dimensiones y que podría atender a la concepción del TAG en el DSM-IV.

La presencia de TAG se estableció mediante una entrevista diagnóstica estructurada basada en los criterios del DSM-IV (APA, 1995) para los trastornos de ansiedad y los del estado de ánimo. Esta entrevista, no publicada, cuestiona, siguiendo el orden establecido en los árboles de decisión del DSM-IV, los diferentes criterios de cada trastorno. En cada trastorno se pregunta inicialmente por los criterios clave y, en caso de respuesta afirmativa, por otras características asociadas al trastorno, tratando de establecer la severidad de la alteración y el cuadro clínico que caracteriza al sujeto. Se incluyeron también cuestiones sobre la posible vinculación del consumo de fármacos o del padecimiento de algún trastorno orgánico con el inicio de los síntomas, para descartar la presencia de trastorno de ansiedad debido a enfermedad médica o a consumo de medicamentos. A 7 personas $(7,2 \%)$ se les asignó el diagnóstico de TAG con esta entrevista.

\section{Procedimiento}

La entrevista diagnóstica y el WAQ fueron aplicados en el contexto de un estudio de prevalencia de los trastornos emocionales en la edad avanzada (Montorio et al., 2001) en el que se seleccionó aleatoriamente desde el censo de una localidad urbana a un $3 \%$ de las personas de más de 65 años, estratificando por edad (65-69, 70-74, 75-79, 80-84, 85-90 o más) y sexo. El porcentaje de participación fue del $58,6 \%$. Las personas de la muestra fueron entrevistadas por licenciados en psicología con formación y experiencia en evaluación gerontológica. Asimismo, el WAQ fue aplicado por el entrevistador, cuyo papel fue estrictamente leer las instrucciones y el contenido de los ítems y consignar las respuestas sin que en ningún caso aportara explicaciones adicionales a lo incluido en el instrumento. Tarjetas con las categorías de respuesta impresas fueron entregadas a las personas para evitar posibles sesgos producidos por deficiencias visuales, facilitar la comprensión y minimizar la posibilidad de deducción o inducción de respuestas (Montorio e Izal, 1999).

\section{RESULTADOS}

En primer lugar, se compararon mediante pruebas $\mathrm{T}$ para muestras independientes las puntuaciones en WAQ entre las 7 personas identificadas con TAG mediante la entrevista diagnóstica y las 90 identificadas como personas sin TAG. Las personas diagnosticadas de TAG (Media = 53,3; DT = $11,6)$ tuvieron puntuaciones significativamente superiores $(\mathrm{t}(95)=7,14 ; \mathrm{p}<0,001$ ) a las personas sin el trastorno (Media = 18.7; DT = 12.4). El tamaño del efecto ( $g$ de Hedges) fue de 2,78 (intervalo confidencial al $95 \%=1,92 / 3,64)$. Dado el reducido tamaño de la muestra clínica, las muestras se compararon también mediante una 
Tabla 1. Sensibilidad, especificidad e índice de Youden para puntuaciones en el WAQ

\begin{tabular}{cccc}
\hline Punto de corte & Sensibilidad & Especificidad & Índice de Youden \\
\hline 38 & $\mathbf{1 , 0 0 0}$ & $\mathbf{0 , 9 1 1}$ & $\mathbf{0 , 9 1 1}$ \\
39 & 0,857 & 0,911 & 0,768 \\
40 & 0,857 & $\mathbf{0 , 9 3 3}$ & 0,790 \\
41 & 0,857 & 0,944 & 0,801 \\
42 & 0,857 & 0,956 & 0,813 \\
43 & 0,714 & 0,967 & $\mathbf{0 , 6 8 1}$ \\
45 & 0,571 & 0,978 & 0,549 \\
47 & 0,571 & 0,989 & 0,560 \\
53 & 0,571 & 1,000 & 0,571 \\
\hline
\end{tabular}

Nota: La fila en negrita es la puntuación que maximiza a la vez la sensibilidad y la especificidad; la ultima puntuación es la que maximiza la especificidad manteniendo una sensibilidad superior a la esperable por azar $(0,500)$.

prueba no paramétrica, obteniéndose asimismo diferencias estadísticamente significativas en la misma dirección (U de Mann-Whitney $=19,5 ; Z=4,1 ; p<0,001$ ). Adicionalmente se analizó la consistencia interna (alpha de Cronbach) del WAQ, obteniéndose una buena fiabilidad $(0,847)$.

En el análisis COR con el WAQ identificando la presencia de TAG la curva dejó por debajo una área de 0,978 ( $\mathrm{p}<0,001$; Intervalo de Confianza al $95 \%=0,948$ / $1,008)$, bajo el supuesto no paramétrico. Una puntuación de 38 (igual o superior) maximizó simultáneamente la sensibilidad $(100 \%)$ y la especificidad $(91,1 \%)$ de la escala para la identificación del TAG (Índice de Youden $=0,911$ ). Por otra parte, un punto de corte de 53 maximizaba la especificidad (100 \%) reteniendo una sensibilidad superior al $50 \%$ que sería esperable por azar $(57,1 \%)$. La puntuación que maximizaba la sensibilidad manteniendo una especificidad superior a la esperable por azar coincide en este caso con el punto de corte que maximiza ambas, 38. En la Tabla 1 se presentan la sensibilidad, la especificidad y el índice de Youden asociados a todas las puntuaciones en el WAQ que se encuentran entre el punto de corte que optimiza simultáneamente sensibilidad y la especificidad y el punto de corte que optimiza la especificidad. Asimismo, en la Figura 1 se representa la curva COR del WAQ para detectar la presencia de TAG.

\section{DISCUSIÓN}

Los resultados de este trabajo ofrecen apoyo adicional a la fiabilidad y validez del Cuestionario de Preocupación y Ansiedad en su uso con personas mayores, al comprobar que puede ser instrumento útil para la identificación del TAG. Así, empleando la técnica COR el área que deja por debajo la curva es elevada $(0,978)$, y presenta muy buenas sensibilidad y especificidad en la identificación en esta edad del trastorno de ansiedad generalizada para una puntuación mayor o igual a 38. Esto permite suge-

\section{Curva COR}

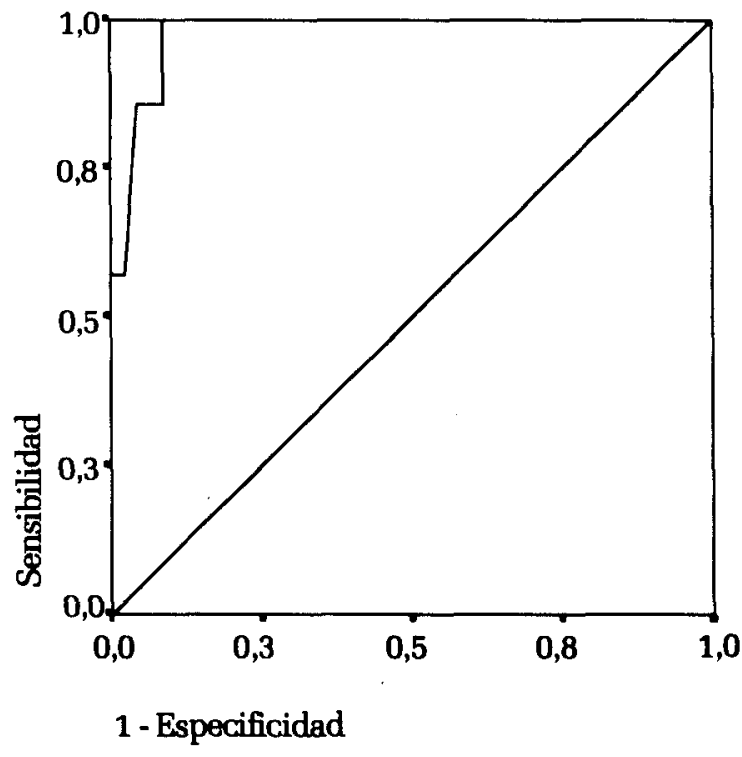

Figura 1. Curva COR para el WAQ prediciendo la presencia de TAG 
rir la utilidad del empleo de esta escala y de los puntos de corte ofrecidos por las curvas COR, como un medio de identificación y cribado del TAG en esta edad. Por supuesto, esta identificación acepta unos márgenes de error superiores a los que deberían ser normales con entrevistas diagnósticas estructuradas aplicadas por evaluadores entrenados. En este sentido, la utilidad de esta escala como instrumentos de cribado para el TAG queda restringida a situaciones en las que, por limitaciones de tiempo, espacio o disponibilidad, no sea posible la realización de tales entrevistas, que, en cualquier caso, deberían seguir a la identificación de un caso positivo.

Algunas limitaciones de este trabajo deberían ser, sin embargo, tomadas en consideración. Por un lado, en el presente estudio no se controló la presencia de otros trastornos, por lo que se desconoce la especificidad y sensibilidad del WAQ para distinguir TAG de depresión u otros trastornos de ansiedad en la edad avanzada. Futuros trabajos deberían intentar aclarar estas cuestiones. Por otro lado, el bajo número de casos clínicos (7) limita el alcance de las conclusiones y sólo permite considerar como preliminar la validación del WAQ como método de cribado para el TAG (aunque la comparación no paramétrica de las puntuaciones del WAQ indica también claras diferencias entre grupos). Futuros trabajos con muestras clínicas más elevadas podrán ofrecer un apoyo más sólido.

Finalmente, como sugieren Fresco et al. (2004), la decisión de qué punto de corte sería adecuado utilizar depende en cada caso de los objetivos que se tengan al usar el instrumento con propósitos de cribado para identificar la presencia de TAG. Así, si el objetivo es identificar a todas las personas con TAG, por ejemplo en el caso de proyectos preventivos, y se desea minimizar el porcentaje de falsos negativos, el punto de corte que optimi- za sólo la sensibilidad (53) sería el adecuado, pero si se desea eludir la presencia de falsos positivos, como por ejemplo para la realización de ensayos clínicos, el punto de corte que maximiza la especificidad (38) sería el más indicado.

\section{REFERENCIAS}

Asociación Psiquiátrica Americana (1995). DSM-IV: Manual diagnóstico y estadístico de los trastornos mentales ( $4^{\mathrm{a}}$ edición). Barcelona: Masson (original de 1994).

Del Ser, T. y Peña-Casanova, J. (1994). Objetivos y métodos de la evaluación de la demencia. In T. del Ser y J. Peña-Casanova (Eds.), Evaluación neuropsicológica y funcional de la demencia (pp. 1-8). Barcelona: JR Prous Editores.

Dugas, M. J., Freeston, M. H., Provencher, M., Lachance, S., Ladouceur, R. y Gosselin, P. (2001). Le Questionnaire sur l'inquiétude et l'anxiété: validation dans des échantillons non cliniques et cliniques. Journal de Thérapie Conportementale et Cognitive, 11, 31-36.

Flint, A.J. (1994). Epidemiology and comorbidity of anxiety disorders in the elderly. American Journal of Psychiatry, 151, 640649.

Fresco, D.M., Mennin, D.S., Heimberg, R.G. y Turk, C.L. (2004). Using the Penn State Worry Questionnaire to identify individuals with generalized anxiety disorder: A receiver operating characteristic analysis. Journal of Behavior Therapy and Experimental Psychiatry, 34, 283-91.

Goldberg, D.P., Bridges, K., Duncan-Jones, P. y Grayson, D. (1987). Dimensions of neuroses seen in primary-care settings. Psychological Medicine, 17, 933-42.

Greenberg, P.E., Sisitsky, T., Kessler, R.C., Finkelstein, S.N., Berndt, E.R., Davidson, J.R.T., Ballenger, J.C. y Fyer, A.J. (1999). The economic burden of anxiety disorders in the 1990s. Journal of Clinical Psychiatry, $60,427-35$.

Himmelfarb, S. y Murrell, S.A. (1984) The prevalence and correlates of anxiety symptoms in older adults. Journal of Psycho- 
logy: Interdisciplinary and Applied, 116, 159-67.

Ibáñez, I., González, M., Fernández-Valdés, A., López-Curbelo, M., Rodríguez, M. y García, C.D. (2000). El Cuestionario de Preocupación y Ansiedad: un instrumento para la evaluación del Trastorno de Ansiedad Generalizada. Ansiedad y Estrés, 6, 203-221.

Lobo, A., Ezquerra, J., Gómez, F.B., Sala, J.M. y Seva, A. (1979). El Mini-Examen Cognoscitivo: Un test sencillo y práctico para detectar alteraciones intelectuales en pacientes médicos. Actas Luso-Españolas de Neurología Psiquiátrica y Científica, 7 , 189-202.

Montorio, I. e Izal, M. (Eds.) (1999). Intervención psicológica en la vejez. Aplicaciones en el ámbito clínico y de la salud. Madrid: Síntesis.

Montorio, I., Nuevo, R., Losada, A. y Márquez, M. (2001). Prevalencia de trastornos de ansiedad y depresión en una muestra de personas mayores residentes en la comunidad. Mapfre Medicina, 12, 19-26.

Nuevo, R., Izal, M., Montorio, I., Losada, A. y Márquez, M. (2003). Dimensiones de contenido de preocupación en población de edad avanzada. Revista de Psicopatología y Psicología Clínica, 8, 223-238.

Nuevo, R., Montorio, I., Izal, M., Ruiz, M.A., Márquez, M. y Losada, A. (artículo enviado para posible publicación). A comparison of the factorial structure of DSM-IV criteria for Generalized Anxiety Disorder between younger and older adults.

Rice, M.E. y Harris, G.T., 1995. Violent recidivism: Assessing predictive validity. Journal of Consulting and Clinical Psychology, 63, 737-748.

Ruscio, A.M., Borkovec, T.D. y Ruscio, J. (2001). A taxometric investigation of the latent structure of worry. Journal of Abnormal Psychology, 110, 413-422.

Shapiro, D. E. (1999). The interpretation of diagnostic tests. Statistical Methods in Medical Research, 8, 113-134.

Stanley, M. A. y Beck, J. G. (2000). Anxiety disorders. Clinical Psychology Review, 20, 731-754.

Wetherell, J. L., Thorp, S. R., Patterson, T. L., Golshan, S., Jeste, D. V. y Gatz, M. (2004). Quality of life in geriatric generalized anxiety disorder: a preliminary investigation. Journal of Psychiatric Research, 38, 305-312.

Youden, W.J. (1950). Index for rating diagnostic test. Cancer, 3, 32-35. 